\title{
Factors that Influence Menstrual Hygiene Management in Adolescent Girls in Mudon Township, Mon State, Myanmar
}

\author{
Nwe Nwe Htun, M.P.H.M. ${ }^{1,2}$, Orapin Laosee, Ph.D. ${ }^{2}$, Cheerawit Rattanapan, Ph.D. ${ }^{2}$ \\ 'People In Need (PIN), INGO, No. 77, Hlaing Myint Mo $1^{\text {st }}$ street, $1^{\text {st }}$ ward, Hlaing Township, Yangon, Myanmar. \\ ${ }^{2}$ ASEAN Institute for Health Development, Mahidol University, Phudthamonthon, Nakhon Pathom 73170, Thailand. \\ Received 14 March 2020 • Revised 16 July 2020 • Accepted 17 July 2020 • Published online 18 December 2020
}

\begin{abstract}
Objective: The study assessed the underlying factors that influence menstrual hygiene management (MHM) in adolescent girls, and how it can have an effect on the girls' education, health and daily life.

Material and Methods: A community-based, quantitative cross-sectional study was conducted through face-to-face interviews, with 410 adolescent girls. Descriptive statistics, chi-square, bivariate and multivariate logistic regression were applied, to describe and predict significant associations and predictors between MHM and its independent variables; such as knowledge, attitudes and practices related to menstruation, and accessibility of MHM facilities.

Results: More than half of the respondents had poor MHM levels. Menstrual knowledge [adjusted odds ratio $(A O R)=$ 2.17, 95\% confidence interval $(\mathrm{Cl}): 1.32-3.56]$, attitude towards menstruation ( $\mathrm{AOR}=1.65,95 \% \mathrm{Cl}: 1.11-2.46)$, menstrual hygiene practices (AOR=2.53, 1.78-3.98) and the accessibility of MHM facilities ( $\mathrm{AOR}=2.44,95 \% \mathrm{Cl}$ : 1.29-4.62) were the factors associated with MHM. Girls with poor MHM levels were 1.1 times more likely to be affected in their daily activities, as compared to those with good MHM levels.

Conclusion: The adolescent girls participating in the study were still unable to manage their menstrual hygiene well, owing to their inadequate knowledge in relation to menstruation, unhealthy menstrual hygiene practices; and the limited accessibility to MHM facilities. Establishment of such correlation necessitates; for instance, setting up a menstrual health education program as well as provision of MHM facilities, will enable the adolescent girls in Mudon Township, Mon State, Myanmar to minimize the negative impacts on their daily lives.
\end{abstract}

Keywords: adolescence, girls, menstrual hygiene management, Myanmar

Contact: Cheerawit Rattanapan, Ph.D.

ASEAN Institute for Health Development, Mahidol University, Phudthamonthon, Nakhon Pathom 73170, Thailand.

E-mail: cheerawit.rat@mahidol.ac.th, cheerawit.rat@mahidol.edu

This is an open access article under the CC BY-NC-ND license

(http://www.jhsmr.org/index.php/jhsmr/about/editorialPolicies\#openAccessPolicy).

J Health Sci Med Res 2021;39(3):207-217 doi: 10.31584/jhsmr.2021778 www.jhsmr.org 


\section{Introduction}

Menstruation is a natural physiological process that is mainly linked with reproduction in girls and women. ${ }^{1}$ Globally, the female population constitutes about $52.0 \%$ of the overall size of the population. About $26.0 \%$ of the global, female population belongs in the reproductive age category. A menstrual cycle takes 21 to 35 days to complete; and the average monthly menstrual flow lasts for two to seven days. ${ }^{2}$ Menstruation is evidence of a healthy reproductive cycle in women of reproductive age, and menstrual hygiene management ( $\mathrm{MHM}$ ) refers to the practices adopted by such menstruating girls and women in the management of hygiene during this menstrual period. $^{3}$

According to the Joint Monitoring Programme of the World Health Organization (WHO) and United Nations Children's Fund (UNICEF): “Women and adolescent girls should use clean menstrual hygiene materials to absorb or collect menstrual blood, which can be changed in privacy as often as necessary for the duration of a menstrual period. Use soap and water for washing the body as required, and having access to safe and convenient facilities to dispose of used menstrual management materials. The basic facts linked to the menstrual cycle, how to manage it with dignity and without discomfort, or fear should be understood". ${ }^{4}$

A study in Taungdwin Gyi, Magway Region, Myanmar showed inadequate knowledge and misconceptions about menstruation and MHM, had negatively affected the girls' confidence to manage their menstrual hygiene. ${ }^{5}$ Many women and girls in low-income countries, including Myanmar, lack access to the materials and facilities that are required to manage their menstrual hygiene. This in turn limited their abilities to carry out many activities, negatively influencing their education, incomes and discharge of domestic responsibilities. ${ }^{6,7}$
Certain misconceptions also prevail in relation to $\mathrm{MHM}$ and practices. These include not taking showers, limiting food intake to certain types of food, and low prevalence of use of soap and water for adequate genital care. ${ }^{8}$ Additionally, some cultural norms and religious beliefs associated with the menstruation cycle, such as remaining house bound, restraining from performing religious activities, and not being able to cook, also influence MHM for such girls and women., ${ }^{9,10}$

Nowadays, in Myanmar, most Women of Reproductive Age (WRA) use sanitary napkins, but some still use a cloth for blood absorption, usually keeping it under their mattresses to help it dry. ${ }^{11}$ In Ye Ye Khin's study (2014), which was carried out among adolescent school girls in Mawlamyine Kyun, Ayawaddy Region Myanmar, almost $98.8 \%$ of respondents used a sanitary pad as a menstrual absorbent, while only $41.4 \%$ changed pads during their school hours. ${ }^{7}$ Irregular, improper and unhygienic replacemnt of the pads can lead to discomfort and irritation; reproductive tract infections (RTI); yeast infections; and urinary tract infections. ${ }^{11-13} \mathrm{~A}$ delay in the treatment of such infections can cause further complications and consequences, such as pelvic inflammatory disease; congenital infection; and chronic renal infection. ${ }^{14}$ The RTIs most closely related to $\mathrm{MHM}$ are bacterial vaginosis and vulvovaginal candidiasis. These infections are passed from the reproductive tract through the materials used to absorb the menstrual blood, or through poor menstrual hygiene. ${ }^{15}$ Therefore, proper MHM can protect girls and women from these infections. ${ }^{16}$

MHM can not only affect physical health, but also education of the WRA. According to an UNICEF (2008) report, about $10.0 \%$ of school girls in Africa remain absent from their schools during their menstruation cycle, and thus miss out on educational outcomes. The major reason for this phenomenon is low access to appropirate menstrual facilities or supplies. Due to pain and fear of leaking eperienced during menstruation, girls may feel 
shame and discomfort, which in turn restricts their active participation in school activities. This situation also exacerbates their inability to remain flexibile to adapt to societal as well as familial interactions. ${ }^{17}$ Support and encouragement extended to the female children to confidently manage their menstrual hygiene, thus helps them to engage in their daily chores and other important life activities. ${ }^{18}$

Adolescents account for about $20.0 \%$ of Myanmar's population, and as they are entering reproductive age knowledge about reproductive health is very important. According to an UNICEF report (2008), early adolescence is classified as being between the ages of 10 and 14 . This is the stage of physical development, in terms of sex organs, and can have and impact on self-concept personality and the accessibility to adequate MHM facilities can be more limited. ${ }^{19,20}$ However, the issue of MHM has been ignored, and policy makers have shown no interest in the Water, Sanitation and Hygiene (WASH) sector in Myanmar. Despite the existence of a budgetary allocation for the national "WASH in Schools" program, with the Ministry of Education as the lead agency, the actual plan has not been chalked out. Some non government organizations, such as the Aung Yadanar Association, are helping to implement this program in an attempt to compliment government efforts. ${ }^{21}$ In addition to this, in the past there only have been a few studies conducted regarding menstrual knowledge and hygiene practices in Myanmar. This situation is further aggravated by the absence of research targeting adolescent girls and their ability to undertake adequate MHM. This study focuses on identifying current abilities of both adolescent girls and the community regarding MHM. The findings and conclusions of this study, in terms of determinants of MHM and its importance in implementing a public health promotion policy and school health programs, will help address the identified gaps in Mudon Township, Mon State, Myanmar.

\section{Material and Methods}

A community-based cross-sectional analytical study design was employed. Structured questionnaires designed for previous studies were adapted. ${ }^{3,6}$ These were used to collect data from all adolescent girls, aged between 9 to 15, living in Mudon Township, and who had already started menarche. The only exclusion criteria were lack of permission from parents, or any physical or mental health conditions preventing participation in the study. The total number of girls in the 9 to 15 years age group was 12,365, and the sample size was estimated by using the Krejcie and Morgan formula.

MHM refers to the (i) articulation, awareness, information and confidence to manage menstruation with safety and dignity, using safe hygienic materials together with (ii) adequate water, agents and spaces for washing and bathing with soap; and (iii) disposal of used menstrual absorbents with privacy and dignity (WHO \& UNICEF). Menstrual knowledge refers to the knowledge of respondents regarding menstruation and $\mathrm{MHM}$. Attitude regarding menstruation and MHM refers to the respondent's feelings and opinions towards menstruation and its hygiene management. Practice of menstrual hygiene refers to the respondent's usual practice for hygiene during menstruation, such as usage of clean, absorbent materials, frequency of changing menstrual materials, disposal method of used materials and use of soap and water to clean hands and body. Accessibility of MHM facilities refers to availability, accessibility and affordability of menstrual absorbent materials, adequate water, soap, space and dust bins to disposed of used materials.

Permission from the local authority, the Township Health Department, was sought first. A pilot study was performed with 30 participants, and the questionnaires were updated based on Kuder-Richardson Formula 20 and Cronbach's coefficient alpha results. Then, the multistage sampling method was applied to select 410 
subjects, and the number of girls was chosen from 4 wards and 38 villages in direct proportion to the number of households. The wards in the town were classified as urban and the villages as rural area. The data were collected through face-to-face interviews, with the girls, after permission from both the guardians and the girls was sought and granted.

The data were analyzed by using Statistical Package for Social Sciences software version 21, with a significance level of 0.05 . Descriptive statistics were used to present the frequencies and percentages of both independent and dependent variables. The total scores were calculated, and mean and median scores were applied as cut of point, respectively; to classify the level of knowledge, attitude, accessibility of MHM facilities and MHM ability. Chi-square tests and binary logistic regression were applied to find the association between each independent and dependent variable. Multivariate logistic regression was used to find the most significant predictors of MHM abilities and their impact.

Confidentiality of the respondents in this study was fully adhered to, with no names of study subjects being divulged, and no personal information about the subjects being made available to anyone for any other purpose. The Ethical Committee of the Institute for Population and Social Research, Mahidol University, approved this study under Certificate No. 2018/02-041, and the Institutional Review Board (IRB) from the Defense Services Medical Research Center, Myanmar, approved the study under Certificate No. IRB/2018/4.

\section{Results}

Descriptive statistics revealed that most of the respondents were Mon and Buddhists in terms of their religious affiliation. About half of the participants (44.1\%) were from urban areas and the remaining (55.9\%) were from rural areas; with the majority being students (83.4\%). It was found that the average age of menarche was $12.33 \pm 1.05$ standard deviation (S.D.) and mothers were their main source of information in concerns to menstruation. Almost all respondents (97.3\%) used sanitary pads to absorb their menstrual blood. The demographic characteristics of the respondents are shown in Table 1.

Table 1 Distribution of respondents by sociodemographic characte

\begin{tabular}{lll}
\hline Sociodemographic characteristics & Number & Percentage \\
\hline Area & & \\
Urban & 181 & 44.1 \\
Rural & 229 & 55.9 \\
Student status & & \\
$\quad$ Yes & 342 & 83.4 \\
No & 68 & 16.6 \\
Source of information about & & \\
menstruation & & \\
Mother & 221 & 53.9 \\
Sisters/aunts & 105 & 25.6 \\
Grandma & 11 & 2.7 \\
Friends & 40 & 9.8 \\
No one & 29 & 7.1 \\
Others (teacher=3, neighbour=1) & 4 & 1.0 \\
Type of sanitary materials & & \\
Sanitary pads & 399 & 97.3 \\
Both sanitary pads and cloths & 10 & 2.4 \\
Not using any sanitary materials & 1 & 0.2 \\
\hline
\end{tabular}

\section{Association between MHM and its independent} variables

The association between MHM and each of the independent variables is shown in Table 2. It revealed that knowledge, attitude, practices and accessibility of $\mathrm{MHM}$ facilities were strongly associated with MHM among seventh independent variables.

\section{Predictors of MHM (multiple logistic regression)}

The variables that were statistically and theoretically significant in the chi-square test, for example menstrual knowledge, menstrual hygiene practices and MHM facilities, were included in this final model. After using multiple logistic regression and the backward Wald method, all of these three variables emerged as the strongest predictors. The results are shown in Table 3. 
Table 2 Association between menstrual hygiene management ability and each independent variable (chi-square \& binary logistic regression)

\begin{tabular}{|c|c|c|c|c|c|c|}
\hline \multirow{3}{*}{ Independent variables } & \multicolumn{4}{|c|}{ Menstrual hygiene management ability } & \multirow{3}{*}{ COR $(95 \% \mathrm{Cl})$} & \multirow{3}{*}{$p$-value } \\
\hline & \multicolumn{2}{|c|}{ Good } & \multicolumn{2}{|c|}{ Low } & & \\
\hline & Number & $\%$ & Number & $\%$ & & \\
\hline \multicolumn{7}{|l|}{ Living area } \\
\hline Urban & 80 & 44.2 & 101 & 55.8 & $1.12(0.75-1.68)$ & 0.581 \\
\hline Rural & 95 & 41.5 & 134 & 58.5 & 1 & \\
\hline \multicolumn{7}{|l|}{ Student status } \\
\hline Yes & 153 & 44.7 & 189 & 55.3 & $1.69(0.98-2.94)$ & 0.059 \\
\hline No & 22 & 32.4 & 46 & 67.6 & 1 & \\
\hline \multicolumn{7}{|l|}{ Knowledge } \\
\hline Poor knowledge level & 128 & 38.9 & 201 & 61.1 & 1 & \\
\hline Good knowledge level & 47 & 58.0 & 34 & 42.0 & $2.17(1.32-3.56)$ & 0.002 \\
\hline \multicolumn{7}{|l|}{ Attitude } \\
\hline Negative attitude & 92 & 37.7 & 152 & 62.3 & 1 & \\
\hline Positive attitude & 83 & 50.0 & 83 & 50.0 & $1.65(1.11-2.46)$ & 0.013 \\
\hline \multicolumn{7}{|l|}{ Menstrual hygiene practices } \\
\hline Poor practice level & 69 & 31.7 & 149 & 68.3 & 1 & \\
\hline Good practice level & 106 & 55.2 & 86 & 44.8 & $2.66(1.78-3.98)$ & $<0.001$ \\
\hline \multicolumn{7}{|c|}{ Accessibility level of MHM facilities } \\
\hline Poor accessibility support & 147 & 40.3 & 218 & 59.7 & 1 & \\
\hline Good accessibility support & 28 & 37.8 & 17 & 62.2 & $2.44(1.29-4.62)$ & 0.005 \\
\hline \multicolumn{7}{|l|}{ Social support level } \\
\hline Poor social support level & 87 & 39.0 & 136 & 61.0 & 1 & \\
\hline Good social support level & 88 & 47.1 & 99 & 52.9 & $1.39(0.94-2.06)$ & 0.101 \\
\hline
\end{tabular}

COR=crude odds ratio, $\mathrm{Cl}=$ confidence interval, $\mathrm{MHM}=$ menstrual hygiene management

Table 3 Final model of multiple regression analysis for predictors of menstrual hygiene management ability

\begin{tabular}{|c|c|c|c|c|}
\hline \multirow{2}{*}{ Independent variables } & \multirow{2}{*}{ Adjust OR } & \multicolumn{2}{|c|}{ 95\% Cl OR } & \multirow{2}{*}{ p-value } \\
\hline & & Lower & Upper & \\
\hline \multicolumn{5}{|l|}{ Knowledge } \\
\hline Poor knowledge level & 1 & & & \\
\hline Good knowledge level & 2.07 & 1.24 & 3.46 & 0.005 \\
\hline \multicolumn{5}{|l|}{ Menstrual hygiene practices } \\
\hline Poor practice level & 1 & & & \\
\hline Good practice level & 2.55 & 1.69 & 3.84 & $<0.001$ \\
\hline \multicolumn{5}{|l|}{ Accessibility of MHM facilities } \\
\hline Poor accessibility level & 1 & & & \\
\hline Good accessibility level & 2.07 & 1.06 & 4.02 & 0.033 \\
\hline
\end{tabular}

$\mathrm{OR}=$ odds ratio, $\mathrm{Cl}=$ confidence interval, $\mathrm{MHM}=$ menstrual hygiene management 
Association between impact on education and each independent variable (multiple logistic regression)

The accessibility level of MHM facilities and attitude towards menstruation were found to be factors significantly associated with an impact on education. This can be seen in Table 4.

\section{Association between impact on daily activities} and each independent variable (multiple logistic regression)

The results showed that the girls with poor MHM could be impacted in their daily activities 3.7 times higher than those who had good MHM (AOR=3.68, 95\% Cl= 2.33-5.82). The results are shown in Table 5.

Table 4 Multiple regression analysis of impact on education and work

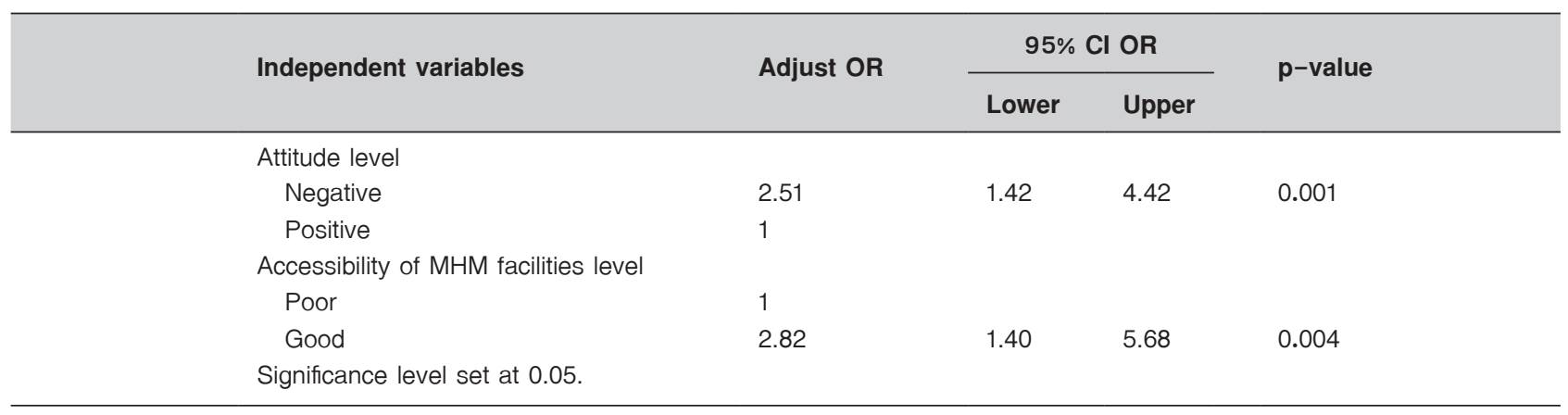

$\mathrm{OR}=\mathrm{odds}$ ratio, $\mathrm{Cl=confidence}$ interval, $\mathrm{MHM}=$ menstrual hygiene management

Table 5 Multiple regression analysis for impact on daily activities

\begin{tabular}{|c|c|c|c|c|}
\hline \multirow{2}{*}{ Independent variables } & \multirow{2}{*}{ Adjust OR } & \multicolumn{2}{|c|}{$95 \% \mathrm{Cl}$ OR } & \multirow{2}{*}{ p-value } \\
\hline & & Lower & Upper & \\
\hline \multicolumn{5}{|l|}{ Knowledge level } \\
\hline Poor & 1.27 & 0.70 & 2.33 & 0.430 \\
\hline Good & 1 & & & \\
\hline \multicolumn{5}{|l|}{ Attitude level } \\
\hline Negative & 4.43 & 2.75 & 7.15 & $<0.001$ \\
\hline Positive & 1 & & & \\
\hline \multicolumn{5}{|l|}{ MHM ability level } \\
\hline Poor & 3.68 & 2.33 & 5.82 & $<0.001$ \\
\hline Good & 1 & & & \\
\hline Significance level set at 0.05 . & & & & \\
\hline
\end{tabular}

OR=odds ratio, $\mathrm{Cl}=$ confidence interval, $\mathrm{MHM}=$ menstrual hygiene management 
Table 6 Multiple regression analysis for impact on health

\begin{tabular}{|c|c|c|c|c|}
\hline \multirow{2}{*}{ Independent variables } & \multirow{2}{*}{ Adjust OR } & \multicolumn{2}{|c|}{ 95\% Cl OR } & \multirow{2}{*}{ p-value } \\
\hline & & Lower & Upper & \\
\hline \multicolumn{5}{|l|}{ Knowledge level } \\
\hline Poor & 1.56 & 0.88 & 2.76 & 0.128 \\
\hline Good & 1 & & & \\
\hline \multicolumn{5}{|l|}{ Attitude level } \\
\hline Negative & 2.36 & 1.51 & 3.68 & $<0.001$ \\
\hline Positive & 1 & & & \\
\hline Significance level set at 0.05 . & & & & \\
\hline
\end{tabular}

OR=odds ratio, $\mathrm{Cl}=$ confidence interval

Association between health impact and each independent variable (multiple logistic regression)

The two independent variables, knowledge regarding MHM and attitudes on menstruation, were analysed to screen the strongest predictors of health impact. The results illustrated that the girls who had a negative attitude towards menstruation were 2.3 times more likely to have their health impacted than the girls who had a positive attitude. The results are shown in Table 6.

\section{Discussion}

In terms of sociodemographic factors, the girls were aged between 10 and 15, and were from both urban and rural areas in equal proportions. The majority were Mon, Buddhist and students. It was found that the mean age of menarche was $12.33 \pm 1.05$ S.D. This was similar to another study that was conducted in Nepal; which showed that the average age of menarche was $13.0 .^{22}$ Almost all of the respondents (97.3\%) used sanitary pads as their main menstrual absorbent materials, and this was similar to a previous study performed in Mawlamyine Township, Mon State. ${ }^{23}$ It is important to note that the girls belonged to a very young age group. Thus, there is a need for the families, community and policy makers to support them in awareness of the menstrual process, and to maximize accessibility to MHM facitlites, including clean absorbent materials.

In regards to menstrual knowledge, only $55.6 \%$ knew that menstruation is controlled by hormones. This was similar to a study in Ethiopia, in which more than half of the girls $(69.3 \%)$ were aware that menstruation is influenced by hormones. ${ }^{24}$ This study also revealed that more than half of the respondents knew that menstruation comes from the uterus (62.4\%). Another study conducted among rural Myanmar women showed that only $23.8 \%$ knew the origin of menstrual blood, while the study in Ethiopia indicated that $64.6 \%$ knew this. ${ }^{5,24}$ Therefore, this study indicates that these adolescent girls did not have adequate knowledge on the process of menstruation and it highlighted the need for rising individual and community awareness to address this.

This study revealed that menstrual knowledge had a strong association with MHM. The girls with a good knowledge level were twice as likely to have good MHM, as compared to those with poor knowledge. According to Girvin (2017), adolescents in Myanmar could not easily obtain reproductive health $(\mathrm{RH})$ information or services if they were not pregnant, because the focus of the limited $\mathrm{RH}$ care available in Myanma is mostly on maternal health. ${ }^{25}$ Although, mothers were their main source of information, 
the results proved that it was neither adequate, nor effective for the girls. The results also showed that sisters, aunts and grandmothers also provided them with menstrual information, but it seems this is still not adequate for effective menstrual awareness.

Menstruation has been regarded as a negative concept in many different settings around the world. ${ }^{26}$ Although, some did not feel shame or have a negative attitude towards it, many menstrual taboos and myths are still present in Mudon; it being a smaller and underdeveloped township. According to Multiple Logistic Regression test, girls who had a positive attitude were twice as likely to be able to manage their menstrual hygiene compared to those who had a negative attitude towards menstruation. This revealed that the girls' acceptance of menstruation as a normal process is important, and the myths and taboos were needed to be minimized by educating them.

The results showed that more than half of the girls felt menstruation disturbs their daily life, although most of them accepted it as a normal physiological process. In another study that assessed the reaction and attitude toward womanhood, menstruation and reproductive events; about one third of the respondents accepted menstruation as natural, however another third were distressed by their menstrual period. ${ }^{27}$ These actions came as a result of certain perceptions of people, based on what they perceived. ${ }^{5}$ Thus, to improve MHM, girls should have proper knowledge as well as a positive attitude towards menstruation and its management.

It can be seen that the practices regarding menstruation were unhealthy, even though almost all of the respondents used sanitary pads. During the data collection period, it was found that the practices of rural girls were unhealthier than those of the urban girls. Most rural girls disposed of their used, absorbent materials in toilets or just threw them away into landfills. Only a small percentage of rural girls discarded them in dustbins or dust bags. More than half of the respondents did not dry them in direct sunlight, because of shame and limited space. About a quarter of the girls washed their hands before and after handling their menstrual absorbents. In another study in Myanmar, more than $90.0 \%$ of participants took baths during their period, but almost none of the participants washed their hands before and after handling their menstrual absorbents. ${ }^{5}$ To promote healthy menstrual hygiene practices among girls, an effective health education program and awareness-raising activities should be carried out by Education, the Public Health department and other relevant authorities.

The findings proved that the accessibility of MHM facilities was strongly associated with the MHM of girls. It was also found that almost all of the girls said they could access private rooms and toilets. They also opined that sanitary pads could be bought easily, and the majority of the girls found them reasonably priced. Nowadays, the price of sanitary products in Myanmar ranges from 600 to $2,000 \mathrm{mmk}(\$ 0.44-\$ 1.48)$. It was also found that there is still no plan in providing sanitary pads in school toilets by school authorities, as most girls went back home to change their pads. According to an UNICEF's report: “Advancing WASH in schools monitoring", Myanmar's coverage is only $23.0 \%$ for sanitation facilities. ${ }^{4}$ More than half of the respondents $(67.8 \%)$ from schools have access to these facilities, and most of the schools can access a water source on-site throughout the year (81.5\%), while about a quarter of the schools provide soap for hand washing. ${ }^{28}$

For girls, there are not enough facilities in a community setting to dispose of used sanitary materials, such as dustbins or dust bags. It seems that they did not think it to be essential, as there are vast landfills in villages to dispose of them. In regards to the MHM facilities in schools, the toilets and WASH facilities could not be observed, because of the school closures during the April 
holidays. It was deduced from the interviews that there were no adequate plastic bags for absorbent materials in the toilets within most of the schools.

The findings also proved that the level of MHM can impact the daily activities of girls, while those who had a negative attitude towards menstruation were impacted in terms of their education and health. Such girls perceived mensturation to be a taboo to be discussed with others, and they did not feel comforatable to participate in class room activities, nor seek support from family and friends for menstrual hygiene related matters. ${ }^{29}$ This confirmed the findings from a study in Indonesia, in which the girls reported that they missed school and had limitations in regard to some activities and performances during menstruation, because of a lack of MHM facilities. ${ }^{22}$ According to the UNICEF's MHM Framework, social support from family, peers and teachers is also vital for menstruating girls. ${ }^{3,4,30}$ A summary report on MHM in South Asia pointed out that girls needed practical and emotional support from peers, men, boys and teachers, who in turn should receive information about MHM; so as to enable them to understand what the girls were experiencing, and provide support if needed. ${ }^{31}$ There have been only a few health awareness sessions regarding $\mathrm{MHM}$ in schools and community settings. However, girls did not mention whether these were held by teachers, school health teams or the public health department.

Myanmar, however, still does not have a functioning national plan for WASH in schools, although there is a budget allocated for this. The guide and toolkits were not accessible and there was no functioning educational management information system to monitor school access to WASH. ${ }^{21}$ In line with the national standards for WASH in schools, more than half of the schools had access to sanitation facilities, with only a quarter of the schools providing soap for hand washing on a daily basis. At the same time it is to be noted that more than a third of these schools can access a water source all year round. ${ }^{21}$ It would be good if the results from this study could draw the attention of education and public health policymakers, with a view of considering support for MHM facilities in both school and community settings.

\section{Conclusion}

Knowledge, attitude and practices regarding menstruation; MHM; and the accessibility of MHM facilities were the determinants of MHM in Mudon Township in Myanmar. Improvement in girls' $\mathrm{MHM}$ and positively affecting their education, work, health and daily activities requires more awareness in regards to menstruation; guidance towards positive attitudes and adoption of healthy menstrual hygiene practices. More importantly, MHM facilities, and a reproductive health education program should be incorporated in school health programs. At the community level, menstrual health awareness-raising activities should be carried out, not only for the young girls themselves, but for guardians as well. Furthermore, the participation of fathers, uncles and brothers, should be regarded as equally important.

\section{Conflict of interest}

None declared.

\section{References}

1. Thakur $\mathrm{H}$, Aronsson A, Bansode S, Lundborg CS, Dalvie S, Faxelid E. Knowledge, practices, and restrictions related to menstruation among young women from low socioeconomic community in Mumbai, India. Front Public Health 2014;2:72.

2. House S, Mahon T, Cavill S. Menstrual hygiene matters: a resource for improving menstrual hygiene around the world. Reprod Health Matters 2013;21:257-9.

3. United Nations Children's Fund. Good practice guilddance of menstrual hygiene maanagement [monograph on the Internet]. New York: United Nations Children's Fund; 2017 [cited 2017 Dec 12]. Available from: https://www.unicef.org/eapro/ MHM_Good_Practice_Guidance_Note_opti.pdf 
4. Dutta D, Badloe C, Lee H, House S. Supporting the rights of girls and women through menstrual hygiene management (MHM) in the East Asia and Pacific Region: realities, progress and opportunities [monograph on the Internet]. Bangkok: United Nations Children's Fund East Asia and Pacific Regional Office; 2016 [cited 2017 Feb 18]. Available from: https://menstrualhygieneday.org/supporting-rightsgirls-women-menstrual-hygiene-management-mhm-eastasia-pacific-region/

5. Nitar W. Menstrual knowledge and practices of rural women in Taungdwingyi Township: a situational analysis. Myanmar Army Health J 2017;4:37-40.

6. Parker HA, Smith AJ, Verdemato T, Webster J, Carter RC. Menstrual management: a neglected aspect of hygiene interventions. Disaster Prev Manag 2014;23:437-54.

7. Khin YY. Awareness and practices related to menstrual hygiene among adolescent schoolgirls in Mawlamyinegyun Township. Yangon: University of Public Health; 2014.

8. Misra P, Upadhyay RP, Sharma V, Anand K, Gupta V. A community-based study of menstrual hygiene practices and willingness to pay for sanitary napkins among women of a rural community in northern India. Natl Med J India 2013;26: 335-7.

9. Guerry E. An assessment of menstrual hygiene practices and absenteeism in Western Uganda. Sheffield: University of Sheffield; 2013.

10. Alam MU, Luby SP, Halder AK, Islam K, Opel A, Shoab AK, et al. Menstrual hygiene management among Bangladeshi adolescent schoolgirls and risk factors affecting school absence: results from a cross-sectional survey. BMJ Open 2017;7:e015508.

11. Schmitt ML, Clatworthy D, Ratnayake R, Klaesener-Metzner N, Roesch E, Wheeler E, Sommer M. Understanding the menstrual hygiene management challenges facing displaced girls and women: findings from qualitative assessments in Myanmar and Lebanon. Confl Health 2017;11:19.

12. Yasmin S, Manna N, Mallik S, Ahmed A, Baisakhi P. Menstrual hygiene among adolescent school students: an in-depth cross-sectional study in an urban community of West Bengal, India. IOSR J Pharm 2013;5:22-6.

13. Haver J, Long JL. Menstrual hygiene management operational guidelines [monograph on the Internet]. Long Island Council:
Save the Children; 2014 [cited 2017 Sep 23]. Available from: https://www.savethechildren.org/content/dam/global/ reports/health-and-nutrition/mens-hyg-mgmt-guide.pdf

14. Juyal R, Kandpal S, Semwal J. Menstrual hygiene and reproductive morbidity in adolescent girls in Dehradun, India. Bangladesh J Med Sci 2014;13:170.

15. Sumpter C, Torondel B. A systematic review of the health and social effects of menstrual hygiene management. PLOS ONE 2013;8:e62004.

16. Ilo $\mathrm{Cl}$, Nwimo IQ, Onwunaka, C. Menstrual hygiene practices and sources of menstrual hygiene information among adolescent secondary school girls in Abakaliki education zone of Ebonyi State. J Educ Pract 2016;7:88-95.

17. Tegegne TK, Sisay MM. Menstrual hygiene management and school absenteeism among female adolescent students in Northeast Ethiopia. BMC Public Health 2014;14:1118.

18. Bingley K. Partnerships review: research for health in humanitarian crises [monograph on the Internet]. London: Elrha; 2019 [cited 2019 Sep 30]. Available from: https://reliefweb.int/sites/ reliefweb.int/files/resources/Partnership-Review-R2HCFINAL.pdf

19. Cobb NJ. Adolescence: continuity, change, and diversity. California: Mayfield Publishing; 1992.

20. Burnet Institute. Adolescent reproductive health in Myanmar: formative research to support the delivery of life-skills education and hygiene improvements through monastic schools in Magway, Myanmar [monograph on the Internet]. Melbourne: Burnet Institute; 2017 [cited 2018 May 24]. Available from:https://www.burnet.edu.au/projects/262_adolescent_ reproductive_health_in_myanmar

21. Wash in school [homepage on the Internet]. Yangon: Aung Yadanar Association [cited 2018 Jun 1]. Available from: http://washinschoolsmapping.com/contact.html

22. Kennedy EWS, Macintyre A, Huggett C, Wheen R. Menstrual hygiene management Indonesia: Understanding practices, determinants and practices, determinants and impacts among adolescents school girls Indonesia [monograph on the Internet]. Yakata: United Nations Children's Fund: Indonesia, Burnet Institute S, Water Aid Australia, Aliansi Rem aja Independen; 2015 [cited 2017 May 20]. Available from: https://www. communityledtotalsanitation.org/sites/communityledtotal sanitation.org/files/MHM_Indonesia.pdf 
23. Bhandari S. Menstrual hygiene practices among adolescence in rural district, Nepal. Nakornphathom Nakhon Pathom: Mahidol University; 2012.

24. Yadanar. Knowledge, practice and associated factors on menstrual hygiene among adolescent school girls in Mawlamine Township. Yangon, Myanmar. Yangon: University of Medicine; 2016.

25. Biruk E, Tefera W, Tadesse N, Sisay A. Assessment of menstrual hygiene management and its determinants among adolescent girls: a cross-sectional study in school adolescent 5 girls in Addis Ababa, Ethiopia. bioRxiv 2018;12:10-16.

26. Girvin SSK, Matthews J. Thai-Burma border reproductive assessment report [monograph on the Internet]. NewYork: Women's Commission for Refugee Women and Children; 2006 [cited 2017 Sep 12]. Available from: https://www.refworld.org/ pdfid/48aa83130.pdf

27. Tan DA, Haththotuwa R, Fraser IS. Cultural aspects and mythologies surrounding menstruation and abnormal uterine bleeding. Best Pract Res Clin Obstet Gynaecol 2017;40:121-33.
28. Wash in school [homepage on the Internet]. Yangon: Aung Yadanar Association [cited 2018 May 24]. Available from: http://washinschoolsmapping.com/5th-annual-virtualconference-on-menstrual-hygiene-management-in-washin-schools.html

29. Nyunt W, Hla HA, Khin MC, Than O, Cho CYM, Lay TH. Reaction and attitude of medical and paramedical lady students towards womanhood, menstruation and reproductive events. Myanmar Med J 2001;45:37-40.

30. Ministry of Drinkig Water and Sanitation, Government of India. Menstrual hygiene management national guidelines [monograph on the Internet]. New Delh: Ministry of Drinkig Water and Sanitation; 2015 [cited 2017 Jan 5]. Available from: https://www. ircwash.org/sites/default/files/mhm_manual-india.pdf

31. United Nations Children's Fund. Menstrual hygiene management in schools in South Asia [monograph on the Internet]. London: Wateraid; 2015 [cited 2017 Feb 5]. Available from: https://washmatters.wateraid.org/sites/g/files/ jkxoof256/files/WA_MHM_SNAPSHOT_INDIA.pdf 